Edited by Allan Beveridge, Femi Oyebode and Rosalind Ramsay

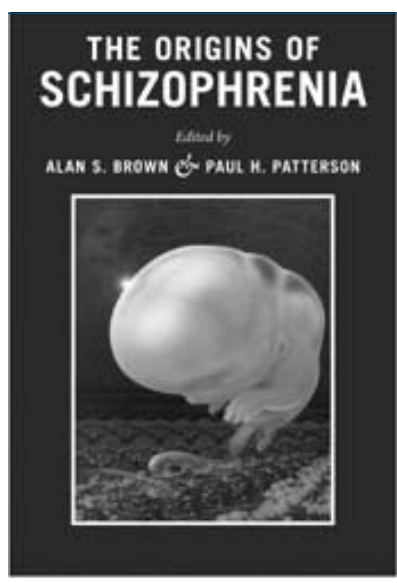

\section{The Origins of Schizophrenia}

Edited by Alan S. Brown \& Paul H. Patterson. Columbia University Press. 2011 £55.00 (hb). 448 pp. ISBN: 9780231151245

Like many researchers, I would guess, I tend to avoid reading, let alone reviewing, books on my subject. My prejudice is that monographs tend to push niche views, whereas multi-author books often lack coherence and the chapters tend to recycle already published data which are usually well out of date by the time of publication. However, I was attracted by the bold title of this one, the reputations of the editors, and by the nagging doubt that during 20 years of digging around in the human genome for the origins of schizophrenia I might benefit from getting a wider perspective. I was not disappointed.

The main focus of the book is on environmental risk factors, although genetic and epigenetic factors are also covered. The book is split into chapters on clinical studies and those on preclinical models and, in the case of maternal infection, prenatal nutrition and maternal stress, there are complementary chapters in both sections. Other putative risk factors that are covered include paternal age, obstetric complications, cannabis use, and vitamin $\mathrm{D}$ deficiency. The chapters are generally well written by experts (the two do not always go together), accessible to the general reader (ditto), and tend to take a balanced view (double ditto). Helpfully, each is prefaced with a list of key concepts and ends with a list of key areas for future research.

The work is framed very strongly within the prevailing paradigm that the origins of schizophrenia are to be found in the interplay between genetic and environmental factors during brain development, and the basis for this view is outlined in an overview chapter. Evidence in favour of a number of the environmental risk factors covered is accumulating thanks to improved epidemiological methodology, but we are still some way from understanding mechanisms. One hope is that findings from new genomics approaches will focus attention on key processes, and early indications are encouraging but too recent to have been integrated into environmental models. A second approach to mechanistic studies is the use of animal models. Although these have been developed, and elegantly so, for factors such as maternal infection, vitamin D deficiency, malnutrition and maternal stress, the problem remains of determining whether neurodevelopmental and behavioural consequences of an environmental manipulation in rodents really model the human disease process. The identification of rare high penetrance mutations in schizophrenia now offers the possibility of developing genetic animal models with high construct validity and the impact of specific environmental manipulation on these might be helpful in this regard.

One striking issue that is not addressed, other than in passing, is that most of the genetic and environmental risk factors for schizophrenia are not specific to the disorder and many, including most of the environmental factors discussed and many of the genetic findings, seem to confer risk to a range of adverse neurodevelopmental outcomes such as intellectual disability, epilepsy, attention-deficit hyperactivity disorder and autism. It seems to me that we should be viewing what we call schizophrenia not as a discrete disorder, but as part of a continuum of liability occurring as a consequence of early brain disruption and that this needs to be taken into account when modelling mechanisms in rodents. This challenges the animal modellers to refine the mapping of their phenotypes on human psychopathology, which is dimensional and crosses disorders, and this in turn requires clinical neuroscientists to understand more fully the neurobiological underpinnings of clinical symptoms and syndromes.

Another omission is the emerging evidence that early childhood maltreatment confers risk to schizophrenia, which is surprising given that this has been modelled in rodents, and the largely neurodevelopmental focus leaves no room for a discussion of possible psychological and social risk factors. These issues notwithstanding, this is an excellent introduction to the origins of schizophrenia, which brings together a large amount of recent work in a readable and critical manner.

Michael Owen MRC Centre for Neuropsychiatric Genetics and Genomics, and Neuroscience and Mental Health Research Institute, Cardiff University, Henry Wellcome Building, Heath Park, Cardiff CF14 4XN, UK. Email: owenmj@cardiff.ac.uk

doi: 10.1192/bjp.bp.111.107300

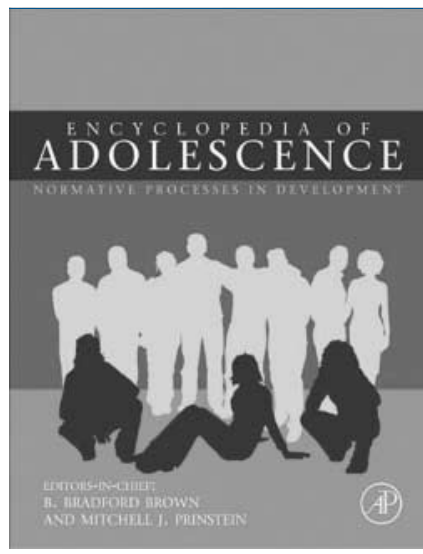

\section{Encyclopedia of Adolescence: Normative Processes in Development}

Edited by B. Bradford Brown \& Mitchell J. Prinstein. Academic Press. 2011. f715.00 (hb). 1294 pp. ISBN: 9780123739155

Almost everything we know about adolescence has come from research conducted during the past quarter of a century. This volume, the first of three, is a distillation of quality research, presented in an engaging style, with extensive cross-referencing, impressive breadth and not a hint of dumbing-down. This volume presents alphabetically organised articles from psychiatry, psychology, biology, history, sociology, public policy expertise, education, geography, literature and anthropology. Within all of these disciplines, there are many definitions of adolescence, yet a characteristic element is that adolescence is a transition whose purpose is equipping children for adulthood.

The point of these narratives is to bring to our attention such diverse topics as child prodigies and how to teach them; neuroplasticity and a more detailed understanding of the selective elimination of brain cells and connections adolescents use least, 